\title{
Recurrent atrial myxoma, right atriotomy, and sinus node dysfunction: A case of interdisciplinary care
}

\author{
Erwin E Argueta MD, Kelly Ratheal MD, Sofia Prieto MD, Ralph Paone MD, \\ Leigh Ann Jenkins MD, Olusegun Oyenuga MD
}

\begin{abstract}
Primary cardiac tumors are rare, and atrial myxomas represent about half of the benign tumors encountered. When found, definitive treatment is surgical resection. Following resection of these tumors, recurrence is possible, and these patients need regular follow-up. In the case of recurrence, repeat surgical intervention is feasible, but the potential for more disruption in atrial anatomy has to be considered. This could contribute to cardiac arrhythmias, and anticipation of these events is necessary to optimize patient care. We present the case of a woman with a recurrent left atrial myxoma who developed sinus node dysfunction after resection and discuss her clinical management.
\end{abstract}

Keywords: Atrial myxoma, atriotomy, sinus node dysfunction

\section{BACKGROUND}

Primary cardiac tumors in adults are rare with an incidence of $0.001-0.03 \%$ (10-300 tumors per 1 million autopsies). ${ }^{1}$ The majority of these tumors are benign, with $50 \%$ being myxomas. ${ }^{2}$ These tumors commonly arise in the left atrium and, while benign, are associated with morbidity due to their ability to cause mechanical obstruction, secrete inflammatory mediators, and cause embolic complications. ${ }^{3}$ The diagnosis of an atrial myxoma is initially suspected by cardiovascular imaging, which is usually transthoracic echocardiography performed for symptoms which suggest cardiac pathology. The cardiac myxoma has characteristic echocardiographic findings that includes its location, attachment to the atrial septum by a stalk, a smooth lobular shape, and gelatinous appearance. ${ }^{4}$ If echocardiographic contrast is given,

Corresponding author: Erwin Argueta Contact Information: Erwin.argueta@ttuhsc.edu DOI: $10.12746 /$ swrcc. v6i25.483 uptake is expected due to the significant vascularization of the tumor. Histology will confirm the diagnosis after resection.

Surgical resection is the treatment of choice and is typically guided by patient symptomatology and/or complications related to the myxoma. The surgical approach for resection is either by right atriotomy with a transseptal approach or left atriotomy. Several studies have shown that right atriotomy with transseptal approach is equivalent to left atriotomy in terms of blood loss and transfusion requirements, extubation times, intensive care length of stay, and postoperative cardiac arrhythmias despite longer aortic cross-clamp times. ${ }^{2,5}$ Atrial arrhythmias or atrioventricular conduction abnormalities can occur in up to $26 \%$ of patients after resection; however, the need for a permanent pacemaker is described in very few cases.

Despite the ability to resect myxomas, recurrence is possible but uncommon. The risk of recurrence has been estimated to be about $13 \%$ and decreases after four years post resection. ${ }^{6}$ The recurrence rates are higher in familial cases. In cases with repeat surgical resection, the increased risk of arrhythmias has 
not yet been well described. We present a case of a recurrent left atrial myxoma which was resected, resulting in persistent arrhythmias that developed in the postoperative period and required pacemaker implantation.

\section{CASE}

A 73-year-old woman initially presented with takotsubo (stress induced) cardiomyopathy and gastroenteritis symptoms. During initial testing, a transthoracic echocardiogram demonstrated a large, highly mobile left atrial mass (Figure 1A). After she was stable, the decision was made to proceed with resection of the mass which would require a sternotomy. Surgical resection was performed via right atriotomy and an interatrial septal incision to access to the left atrium. Here the mass was attached to interatrial septum and completely excised. After completing left atrial and left ventricular exploration to exclude any residual tumor, the interatrial septum and the initial right atrium incisions were sutured. The mass was soft and myxoid of $4 \times 3 \times 1.5 \mathrm{~cm}$ in its largest dimensions. The postoperative period was complicated by an ischemic frontal lobe cerebrovascular accident and paroxysmal atrial fibrillation which converted to sinus rhythm spontaneously.

Although the echocardiogram performed five months after surgical resection revealed no evidence of a myxoma, a repeat study at 18 months after resection revealed a recurrent left atrial myxoma (Figure 1B) The decision was then made to remove the tumor due to her stroke risk. The surgical approach again involved a right atriotomy followed by an interatrial septum incision. However, now the superior portion of the interatrial septum was excised together with the pedunculated myxoma attached to it. Subsequently the interatrial septum was repaired without the need for a prosthetic patch. Images are shown in Figure $2 \mathrm{~A}$ and $\mathrm{B}$. Prior to surgery, her ECG showed sinus rhythm. In the postoperative period she had adequate wound and respiratory recovery, but she required epicardial atrial pacing for 72 hours due to sinus arrest. She resumed sinus node activity on postoperative day four (Figure 3) but developed atrial flutter on day five

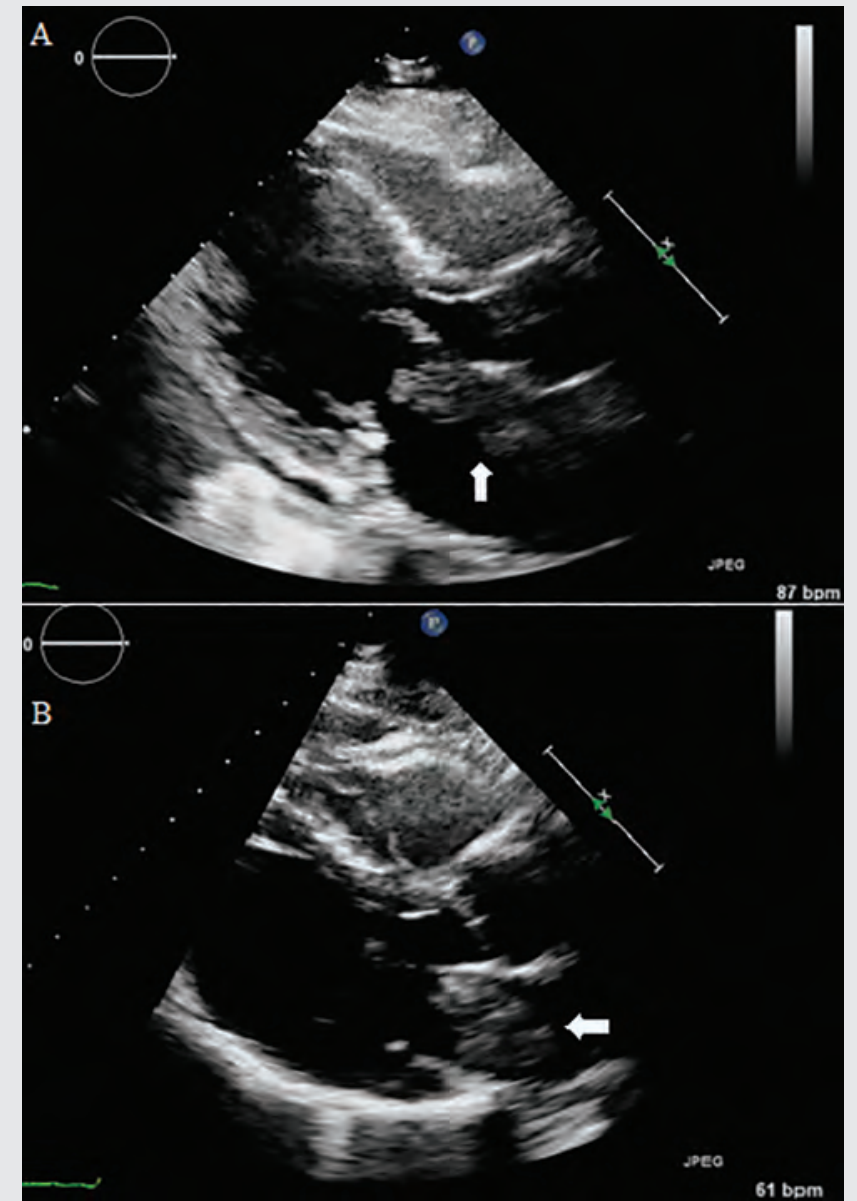

Figure1 Transthoracic echocardiography reveals a large atrial myxoma (arrow) inside the left atrium in a PLAX ${ }^{*}$ view on initial diagnosis (A). Repeat echocardiography 18 months after initial myxoma resection $(B)$.

(Figure 4). Due to her symptoms and poor rate control with medical therapy, she required synchronized cardioversion. After cardioversion, she again developed sinus arrest which was followed by an ectopic atrial rhythm (Figure 5). Her need for an AV nodal blocking agent, persistent sinus node suppression with chronotropic incompetence, and concern for other episodes of post conversion pause ultimately influenced the decision to proceed with dual chamber pacemaker implantation. After electrical stability was established, she was discharged home with outpatient follow up. 

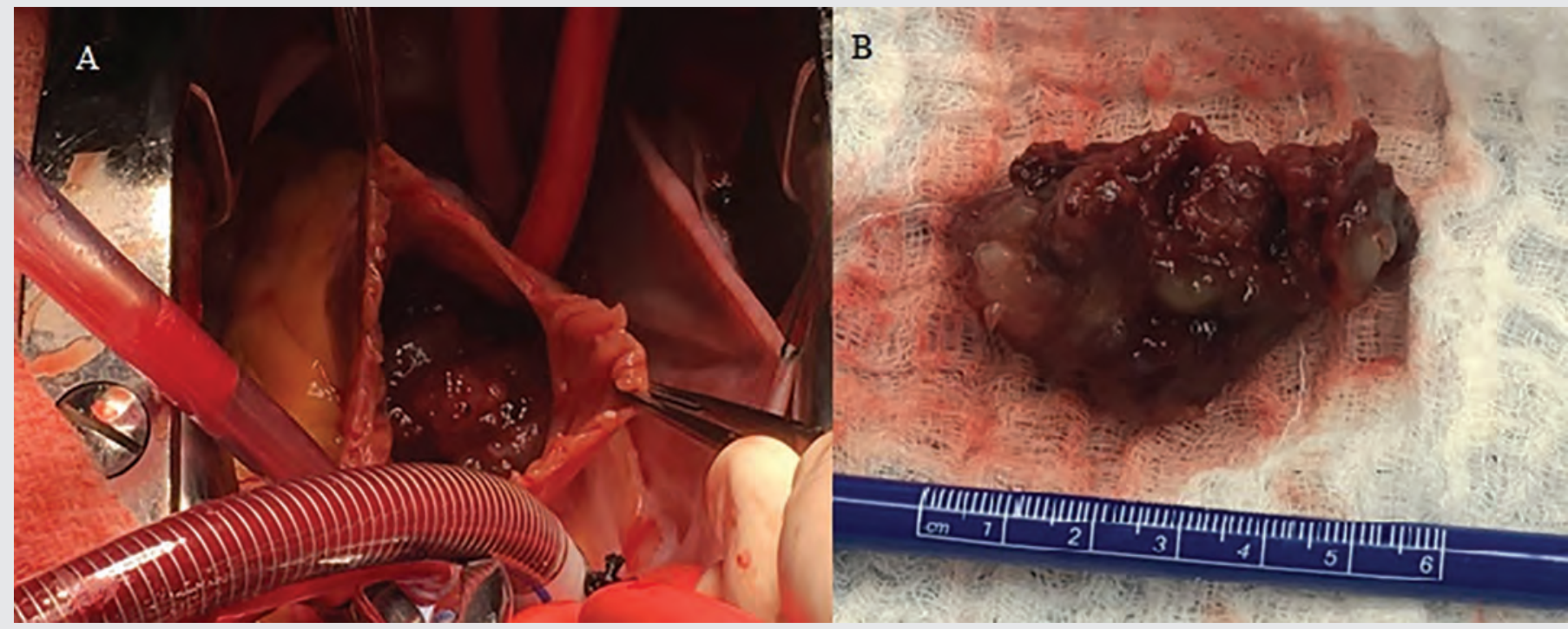

Figure2 Left atrial myxoma inside the atrium prior to resection (A), and after (B).

At one year, the patient remained stable with no identified mass on transthoracic echocardiography.

\section{DIscussion}

This case exemplifies a classic presentation of an atrial myxoma in a female patient just past her sixth decade of life. Rhythm disturbances after right atriotomy, including immediate and late (scar mediated)

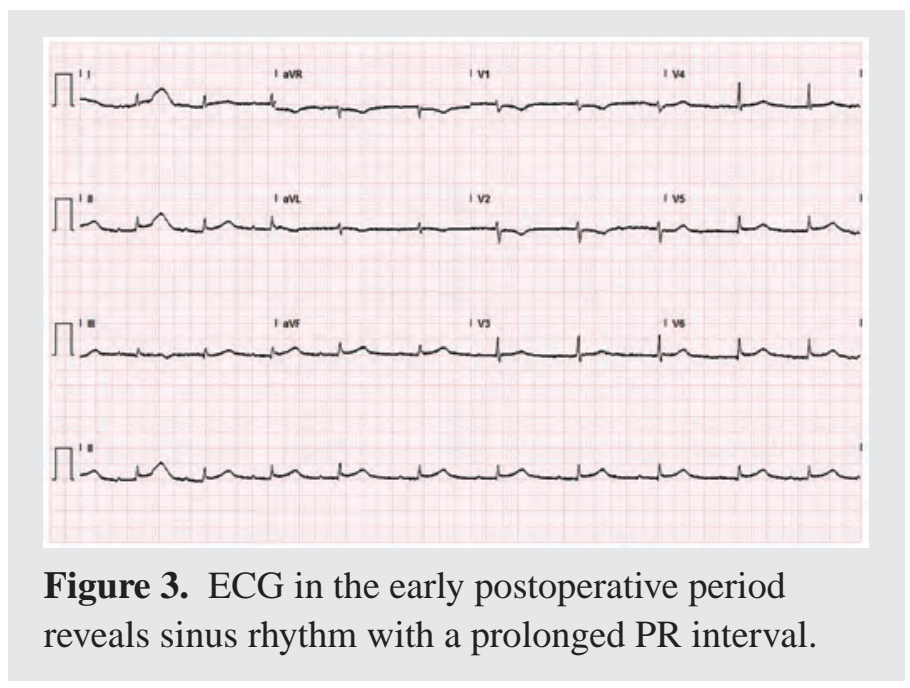

tachyarrhythmias, have been well documented. The patient's re-operation was associated with a more aggressive excision increasing the risk of bradyarrhythmia due to sinus node or AV node conduction damage. It is important to note that during the repeat surgical intervention the right atrium was incised again, which is the anatomic structure where the sinus node is located. In addition, on the second surgical intervention part of the superior interatrial septum was

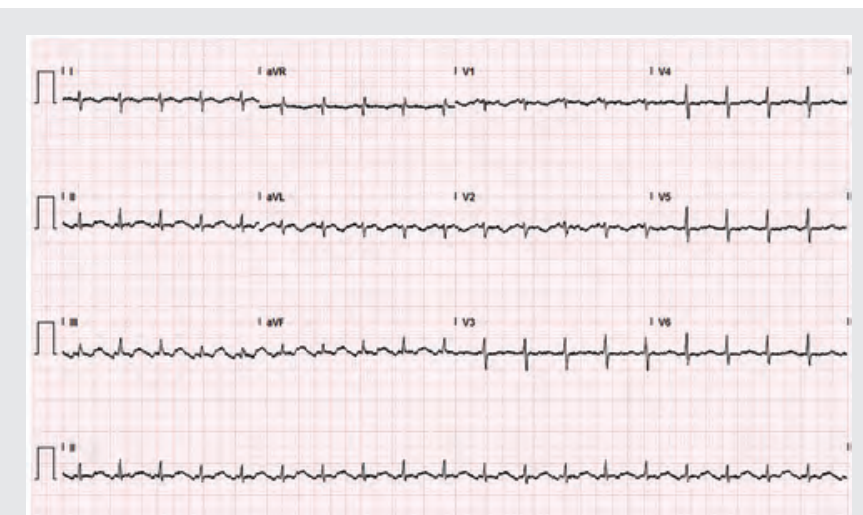

Figure4. ECG demonstrates atrial flutter on postoperative day five. 


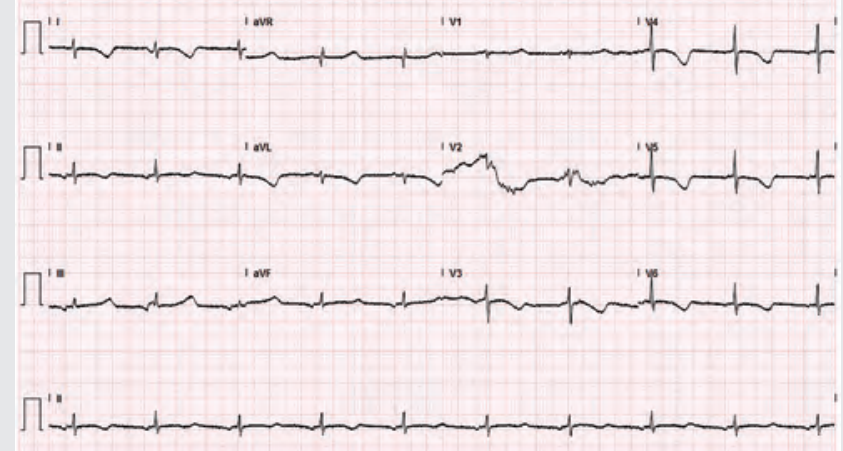

Figure 5. Ectopic atrial rhythm, the $P$ waves have changed in morphology when compared to the patient's baseline rhythm.

excised, and the septum was subsequently repaired. While difficult to know if any atrial electrical conduction pathways were damaged, the Bachmann's bundle provides communication between the atria.

Atrial arrhythmias or atrioventricular conduction abnormalities after myxoma resection are reported to be the most common complication, both in the immediate postoperative period and at long-term follow up. ${ }^{7}$ While post resection arrhythmias occur in $26 \%$ of patients, the need for permanent pacemaker is very rare (about 2.6\%). ${ }^{8}$ These statistics are not specific to the type of surgical approach taken, and there is little information about arrhythmia rates after a left atrial approach compared to a right atrial approach. Furthermore, there are few evidence-based medical recommendations regarding management of arrhythmias after repeat myxoma resection.

The rhythm our patient had after cardioversion suggested sinus node dysfunction likely related to the repeat surgical intervention. During the second resection, more tissue was removed due to concern for recurrence. Additional scar tissue could provide a substrate to perpetuate supraventricular arrhythmias. With the above mentioned electrocardiographic findings after surgery, her history of supraventricular arrhythmias, and the need to initiate rate control therapy, it was decided to proceed with a dual chamber pacemaker implantation secondary to sinus node dysfunction.

\section{Conclusion}

Atrial myxoma recurrence is uncommon, and there is limited information on the management and typical clinical course. While arrhythmias may occur after myxoma resection, few patients require a permanent pacemaker. In addition to the multidisciplinary care needed, this case highlights two important points. First, surveillance for myxoma recurrence is necessary. Second, it is important for providers to be aware of surgically related changes to the atrial cardiac anatomy that can result in electrical conduction alterations expressed as tachy- or bradyarrhythmias in the early postoperative period. By recognizing the increased potential for arrhythmia, it is possible to make timely interventions to optimize long term outcome goals.

Article citation: Argueta EE, Ratheal K, Prieto S, Paone $\mathrm{R}$, Jenkins LA, Oyenuga O. Recurrent atrial myxoma, right atriotomy, and sinus node dysfunction: a case of interdisciplinary care. The Southwest Respiratory and Critical Care Chronicles 2018;6(23):42-46

From: Division of Cardiovascular Diseases, Department of Internal Medicine (EEA, KR, SP, LAJ, OO); Division of Cardiothoracic Surgery (RP), Texas Tech University Health Sciences Center, Lubbock

Submitted: 4/22/2018

Accepted: 6/15/2018

Reviewers: Anurag Singh MD, Aliakbar Arvandi MD Conflicts of interest: none

This work is licensed under a Creative Commons Attribution-ShareAlike 4.0 International License

\section{REFERENCES}

1. Reynen K. Frequency of primary tumors of the heart. A m J Cardiol 1996;77:107.

2. Elbardissi AW, Dearani JA, Daly RC, et al. Survival after resection of primary cardiac tumors: a 48-year experience. Circulation 2008;118:S7-15. 
3. Vaideeswar P, G upta R, M ishra P, et al. A typical cardiac myxomas: a clinicopathologic analysis and their comparison to 64 typical myxomas. Cardiovasc Pathol 2012;21:180-7.

4. Lee VH, Connolly HM, Brown RD, Jr. Central nervous system manifestations of cardiac myxoma. Arch Neurol 2007;64:1115-20.

5. Hatemi $A C$, Gursoy $M$, Tongut $A$, et al. L eft atriotomy versus right atriotomy trans-septal approach for left atrial myxoma. J Int M ed Res 2010;38:276-81.
6. Pinede L, D uhaut $P, L$ oire R. Clinical presentation of left atrial cardiac myxoma. A series of 112 consecutive cases. Medicine (Baltimore) 2001;80:159-72.

7. Scrofani R, Carro C, Villa L, et al. [Cardiac myxoma: surgical results and 15-year clinical follow-up]. Ital Heart J Suppl 2002;3:753-8.

8. Wang Z, Chen S, Zhu M, et al. Risk prediction for emboli and recurrence of primary cardiac myxomas after resection. J Cardiothorac Surg 2016;11:22. 